\title{
A Colossal Rhinolith
}

https://doi.org/10.47210/bjohns.2021.v29i1.352

Neha Shakrawal, ${ }^{1}$ Bikram Choudhury, ${ }^{1}$ Kapil Soni, ${ }^{1}$ Darwin Kaushal ${ }^{1}$

\section{ABSTRACT}

Introduction

Rhinolith is a calcic deposition formed by mineralization of salts in an impacted nasal foreign body. It is a benign condition that can be troublesome owing to its size and extent of impact.

Case Report

A 35-year-old man with a history of right nasal obstruction with occasional foul-smelling discharge and right eye pain since ten years was diagnosed with rhinolith. During its removal, it was three times bigger than what could be visualized in nasal endoscopy.

Conclusion

Proper history, examination, and a high suspicion can clinch the diagnosis in almost all cases. A rigid diagnostic nasendoscopy is an important tool in the diagnosis. The treatment of choice is endoscopic removal under local or general anaesthesia.

$\underline{\text { Keywords }}$

Rhinolith; Foreign Bodies; Nose; Nasal Obstruction

$\mathrm{T}$

The Rhinolith gets its name by a conjoint of two separate words i.e. "Rhino" and "Lith" implying a "stone in the nose". Rhinolithiasis had been described by Bartholin way back in 1654. It is an entity formed by gradual deposition and coating of different salts of calcium and magnesium over an endogenous or exogenous nidus in the nasal cavity. ${ }^{1} \mathrm{~A}$ variety of exogenous nidus may be seen ranging from small to large size i.e. paper, stone, grain, seed, grass, glass, wood, rubber medications etc. These are the most common cause of rhinoliths seen. Endogenous nidus can be dried up secretions, blood clots, pus, mucosa necrotic debris, hair follicles, or tooth remnants. ${ }^{2}$

The most common location is floor of nose, at the junction of anterior and posterior nares. Normally they sit anteriorly but due to normal physiological action they tend to shift posteriorly and retain a more favourable position. Local inflammatory reaction leads to a

1 - Department of Otorhinolaryngology, All India Institute of Medical Sciences, Jodhpur

\section{Corresponding author:}

Dr Bikram Choudhury

email: comatosebuddha@gmail.com deposition of salts of calcium phosphate, magnesium, calcium carbonate, and aluminium. All these changes around focal nidus make it hard in texture and the size of the rhinolith increases gradually.

The type, size and duration of the rhinolith lead to multiple types of presentations. The insidi-ousness and gradual development leads to the symptoms gradually developing years after the process begins, causing persistent or recurrent nasal infections. The most common clinical pic-ture is that of a unilateral nasal obstruction and foul smelling discharge. The patient may pre-sent with symptoms such as cacosmia, epistaxis, headache, facial pain and even epiphora.

If a routine anterior rhinoscopy is unable to make a diagnosis, endoscopic examination can lead to diagnosis. Radiology also plays an important role. If the rhinolith is highly radiopaque they can be identified in conventional $\mathrm{x}$-rays. If rhinolith is small or less radio opaque, then $\mathrm{CT}$ can be helpful in localizing it.

Early diagnosis is needed to prevent late sequelae. The gold standard of management of these rhinoliths is endoscopic removal. The rhinolith size and extent helps us in deciding on the approach. ${ }^{3}$ A more impacted and larger rhinolith should be removed under sedation as 


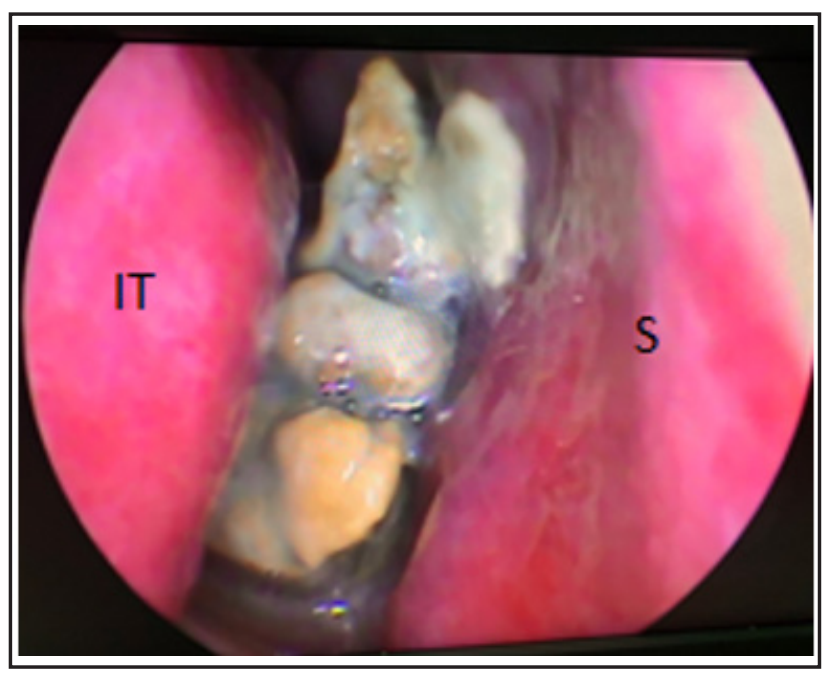

Fig.1. Zero degree nasendoscopy showing grayishyellow rhinolith in the right nasal cavity.

they are extremely painful. In this report we describe a giant rhinolith, which was likely caused by a nidus of a foreign body. We also discuss the surgical management that was performed to cure the patient.

\section{Case Report}

A 35 year old healthy man reported to the Department of Otorhinolaryngology. He had right nasal obstruction with occasional foul smelling discharge and right eye pain since 10 years. There was no history of foreign body insertion or nasal trauma. Treatment till date had been chiefly antibiotics and nasal decongestants intermittently but the patient had no significant re-lief.

Anterior rhinoscopy revealed secretions in right nasal cavity. On performing rigid nasendoscopy on right side, after suctioning out the secretions, an irregular yellowish gray friable mass was visualized with contact bleeding at the level of half way of inferior turbinate. It was extending superiorly up to middle meatus. (Fig.1)

Septum was also deviated towards left side due to the mass effect. The left side nasal cavity was patent with no abnormality. Patient was advised for x-ray which was suggestive of radiopaque mass in right side floor of nose. (Fig.2) Rest of the sinuses was normal. The history, clinical findings and radiology suggested that

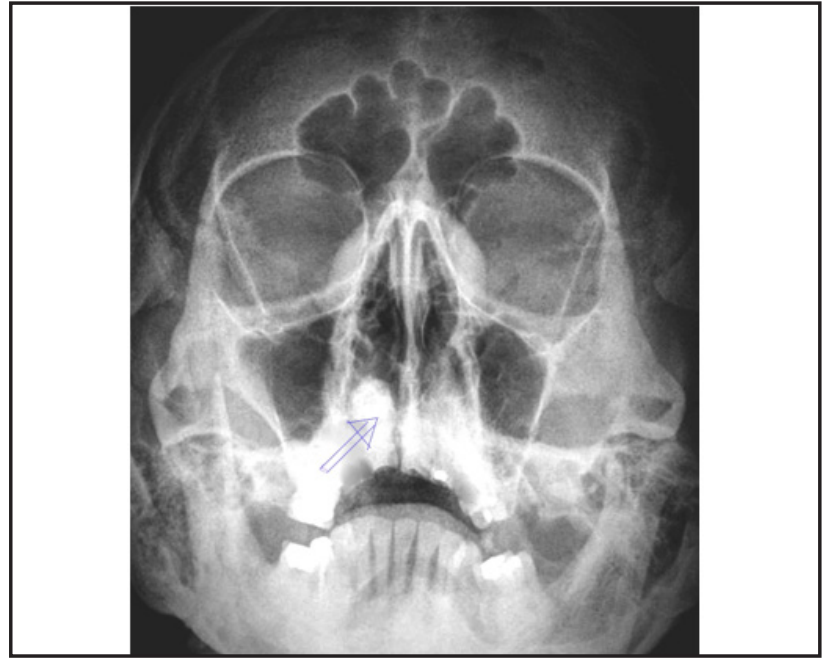

Fig.2. X ray PNS showing radio-opaque mass in floor of right nasal cavity.

the cause of the unilateral nasal obstruction was a rhinolith.

Endoscopic removal of rhinolith was planned under local anesthesia. To our surprise it was three times bigger than what could be visualized in nasal endoscopy. Ideally a large and friable rhinolith should be fragmented and removed in piecemeal. It was occupying whole of the right nasal cavity. Minimal bleeding was also encountered which was addressed with local anesthetic and adrenaline patties. The rhinolith was removed in piecemeal. (Fig.3) Inferior and middle tur-binate were found atrophic after complete removal. Middle meatus was found free of any debris or collection. Nasopharynx was inspected and was found to be normal. Merocel ${ }^{\circledR}$ was utilized to pack the right nasal cavity and a nasal bolster was applied. Post-operative period was unevent-ful. Pack was removed after 24 hours and he was advised saline nasal douching. The specimen was handed over to the patient and it could not be sent for the histopathology. Patient is doing fine at 16 months of follow-up.

\section{Discussion}

Rhinolith is a rare entity in this era of increasing awareness and hygienic habits. They are de-fined as nasal calculi formed by calcareous deposition over 


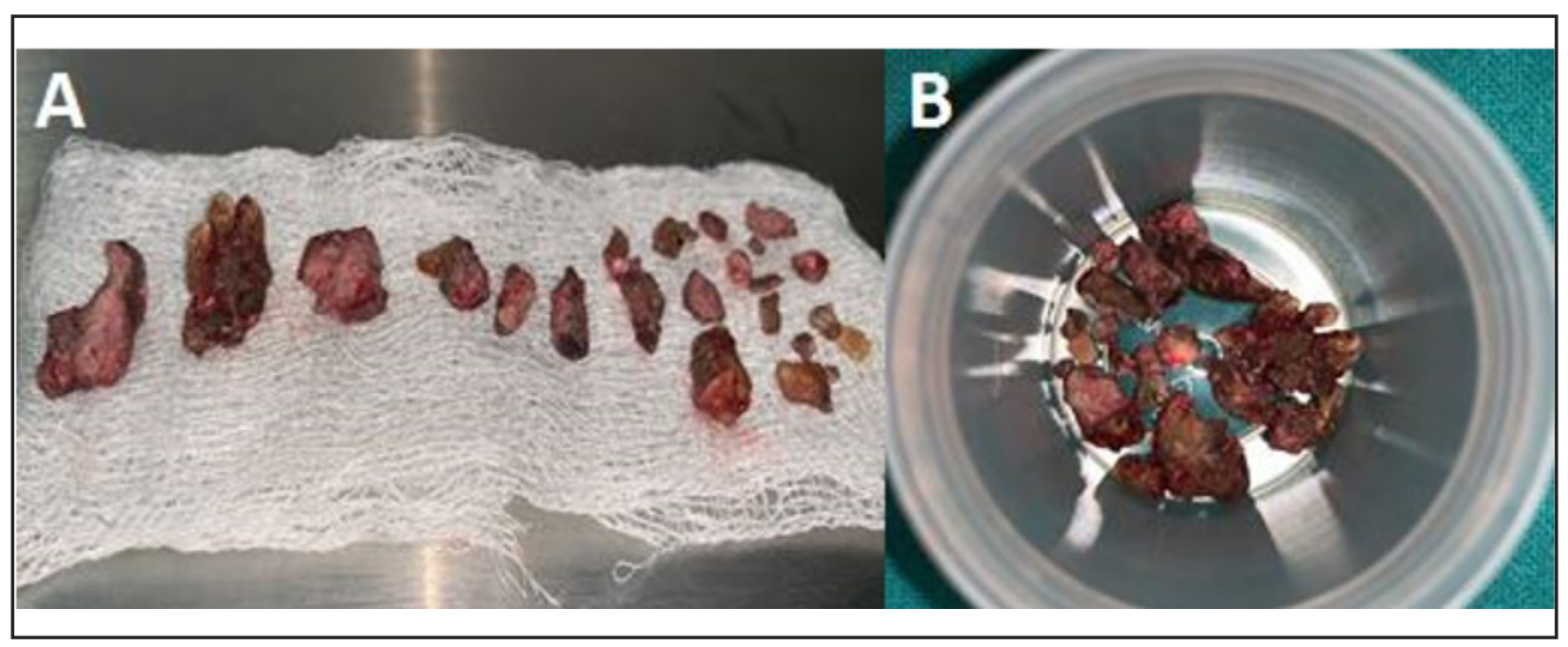

Fig.3. (A) Rhinolith retrieved in piecemeal (B) Total weight of $20 \mathrm{gms}$.

retained intranasal foreign bodies. Polson in 1943 reported a giant rhinolith of around a size of pinecone. It's removal was wit-nessed and documented by a colleague. ${ }^{4}$

As mentioned above, it is unclear, what is the pathogenesis of a rhinolith. It is suspected that a retained foreign body acts as a foci or nidus over which inflammatory process sets in and depo-sition of mineral salt occurs. ${ }^{2}$ Axmann was the first to do a chemical analysis of a rhinolith and determined that a rhinolith consists of $90 \%$ inorganic material and $10 \%$ organic substances. ${ }^{5}$ The mode of entry of foreign body can be anterior or posterior depending upon the act of insertion. Most common site is anterior but due to coughing, eating and talking they lodge posteriorly. Depending upon the characteristics of any foreign body- the foreign bodies are classified to be True i.e. endogenous or False i.e. exogenous. ${ }^{6}$ A rare case of opioma has been reported to cause rhinolith formation. ${ }^{7}$ It is more commonly seen in females. ${ }^{4}$ The age ranges from 3 to 76 years with peak incidence between 4 th to 5 th decades. Rhinolith are mostly single and unilateral. The size can vary from few $\mathrm{mm}$ to cms. They often extend to occupy the whole of the nasal cavity and usually take its shape. There is literature available of a rhinolith appearing like the egg of a hen weighing 115 gms. ${ }^{6}$ The most common site of a rhinolith is at inferior turbinate, abutting the nasal septum..$^{5}$

In our case, we assume the possible etiology could be a retained foreign body accidentally in-serted in the childhood. The patient does not gave history regarding recurrent allergies, epistaxis or sinusitis like symptoms. So it is unlikely because of retained secretions or blood clots.

Some common alternate differential diagnosis causing unilateral nasal obstruction are calcified polyps, odontomes, granulomas, granulomatous dissemination, sequestration following osteomyelitis, osteomas, calcified otogenic cysts, chondrosarcoma, osteosarcomas and sinonasal ma-lignancies. ${ }^{8}$ Other possible etiologies of unilateral nasal obstruction can be physiological, structural or pathological. It can be partial or complete, intermittent or continuous. Physiological causes include blockage at intervals due to nasal cycle. Structural causes include deviated nasal septum, hypertrophied inferior turbinate or choanal atresia. Pathological causes include sinusitis, polyps, neoplasms or granulomatous diseases.

Diagnosis is usually done by anterior rhinoscopy and confirmed by using a diagnostic nasal en-doscopy which is minimally invasive procedure. It also avoids exposure to any radiation. So, preferably it should be done prior to radiology. If necessary, an orthopantomogram or $\mathrm{x}$-ray of the paranasal sinus, or a non-contrast computed 
tomographic scan may be done. In 1900, MacIntyre described the radiology of rhinolith for the first time. The features were mixed radiopaque and radiolucent mass which are arranged in a concentric circle or lamellas. ${ }^{9}$ In case of small and impacted rhinolith, computed tomographic scan plays an important role as it can localize the extent and can warrant any complication. Long standing cases can lead to multiple complications including serous otitis media, sinusitis (of either bacterial or fungal origin), perforation of the septum or palate or, chronic dacryocystitis. ${ }^{9}$

The ideal management is surgical removal either in local or general anesthesia depending upon the nature and extent of rhinolith. Post-operative douching is important to prevent further crust-ing and reduce complications.

\section{Conclusion}

Although rhinolith is a rare entity but a good clinical history, physical examination and a high suspicion can clinch an early diagnosis and prevent complications. Rigid diagnostic nasendoscopy plays an utmost role in the management. Treatment of choice is removal of rhinolith keep-ing the structural and functional integrity of the nose intact.

\section{References}

1. Ji-Chang H, Chih-Feng T, Ka-Wo L, Kuen-Yao H, Wen-Rei K, Feng WL. Giant rhinolith: a case report. Kaohsiung J Med Sci. $2005 ; 21: 582-4$

2. Carder HM, Hill JJ. Asymptomatic rhinolith: A brief review of the literature and case report. Laryngoscope 1996; 76:524-30

3. Dib GC, Tangerina RP, Abreu CEC, de Paula Santos R, Gregório LC. Rhinolithiasis as cause of oronasal fistula. Braz J Otothinolaryngol. 2005;71(1):101-3

4. Polson CJ. On rhinoliths. J Laryngol Otol. 1943;58:79-116

5. Marfatia PT. Rhinolith. A brief review of the literature and a case report. Postgraduate Medical Journal 1968;44:478-9

6. Langlais RP, Langland OE, Nortje CJ. Diagnostic imaging of the jaws. Chapter 19 "Soft tissue radiopacities". Williams and Wilkins, Baltimore 1995; 630-31

7. Ghanbari H, Farhadi M, Daneshi A. Report of an unusual cause of rhinolithiasis: An 'opioma'. Ear Nose Throat J. 2007; 86(1):48-9

8. Chaker AC, Schwartz GS, Kole GL. Bilateral Rhinolithiasis Ear Nose \& Throat Journal 1978; 57: 15-26

9. Stammberger H, Jakse R, Beaufort F. Aspergillosis of the paranasal sinuses: $\mathrm{X}$ ray diagnosis, histopathology, and clinical aspects. Ann Otol Rhinol Laryngol. 1984; 93:251-6. 